\title{
Clinical trial of oral administration of Bifidobacterium longum in dogs with atopic dermatitis
}

\author{
Kang-Il Lee ${ }^{1, \dagger}$, Taesik Yun ${ }^{1, \dagger}$, Junsang Ham ${ }^{2}$, Wan-Kyu Lee ${ }^{3}$, Ji-Houn Kang ${ }^{1}$, \\ Mhan-Pyo Yang', Byeong-Teck Kang ${ }^{1, *}$ \\ ${ }^{1}$ Laboratory of Veterinary Internal Medicine, Veterinary Teaching Hospital, College of Veterinary Medicine, \\ Chungbuk National University, Cheongju 28644, Korea \\ ${ }^{2}$ National Institute of Animal Science, Rural Development Administration, Jeonju 54875, Korea \\ ${ }^{3}$ Laboratory of Veterinary Bacteriology and Infectious Diseases, College of Veterinary Medicine, Chungbuk National University, \\ Cheongju 28644, Korea
}

\begin{abstract}
This study assessed the effects of probiotics in canine atopic dermatitis (AD). We enrolled 11 client-owned dogs with AD and randomly allocated them to two groups. The probiotics group $(\mathrm{n}=7)$ was prescribed with Bifidobacterium longum while the control group $(n=4)$ received a placebo powder once a day for 12 weeks. In both groups, the degree of skin lesions was evaluated based on the Canine Atopic Dermatitis Extent and Severity Index (CADESI)-4. We also measured the transepidermal water loss (TEWL). We assessed pruritus severity using the pruritus visual analog scale (PVAS). Alteration of consumed drug doses was converted into medication scores. All the evaluation indices were surveyed every 4 weeks. In the probiotics group, there was a significant decrease in the CADESI-4 score at 4, 8 , and 12 weeks compared to that of the baseline score $(p<0.05)$. There was no significant difference in TEWL, PVAS, and medication score at each time point and between groups. Although these results showed that Bifidobacterium longum did not reduce pruritus, TEWL, and the dosage of drugs for canine AD, it was effective in improving skin lesions, therefore, probiotics could be considered in canine AD with severe skin symptoms.
\end{abstract}

Keywords: Atopic dermatitis, Bifidobacterium longum, dogs, probiotics

*Corresponding author

Byeong-Teck Kang

Laboratory of Veterinary Internal Medicine,

Veterinary Teaching Hospital, College of

Veterinary Medicine, Chungbuk National

University, 1 Chungdae-ro, Seowon-gu,

Cheongju 28644, Korea

Tel: $+82-43-261-3744$

Fax: +82-43-267-2595

E-mail: kangbt@chungbuk.ac.kr

The first two authors contributed equally to this work.

ORCID:

Kang-Il Lee

https://orcid.org/0000-0002-6597-9948

Taesik Yun

https://orcid.org/0000-0003-1372-4430

Junsang Ham

https://orcid.org/0000-0003-4966-6631

Wan-Kyu Lee

https://orcid.org/0000-0001-5087-6359

Ji-Houn Kang

https://orcid.org/0000-0001-8536-3027

Mhan-Pyo Yang

https://orcid.org/0000-0002-8043-0152

Byeong-Teck Kang

https://orcid.org/0000-0002-4471-4342

Conflict of Interest

The authors declare no conflicts of interest.

Received: September 18, 2019

Revised: December 18, 2019

Accepted: January 31, 2020

\section{Introduction}

Canine atopic dermatitis (AD) refers to genetically predisposed allergic dermatosis that causes chronic pruritus associated with exposure to numerous types of environmental allergens [1]. It is generally associated with typical clinical signs and the production of allergen-specific immunoglobulin E (IgE) antibodies [2]. In past decades, the prevalence of canine AD has increased [3]. Various strategies have been proposed to alleviate the clinical signs of canine AD including avoidance of allergens, application of anti-inflammatory drugs, and allergen-specific immunotherapy [4]. Allergen-specific immunotherapy is not a common treatment for canine $\mathrm{AD}$ because it requires the long-term subcutaneous or sublingual administration of allergens [4]. On the other hand, administration of anti-inflammatory and anti-pruritus drugs including topical/ systemic glucocorticoids, ciclosporin, and oclacitinib is the main treatment option for canine AD. However, since long-term or high-dose use of these drugs has been associated with some adverse effects, concomitantly, a variety of adjunctive methods in the treatment of canine AD have been attempted to reduce the dosage of these drugs.

Studies have reported that probiotics, which are one of the adjunctive treatments and include specific microorganisms, could be effective against canine AD [5-7]. The effect of bacteria that produce lactate on canine $\mathrm{AD}$ has been described in the International Committee on Allergic Diseases of Animals (ICADA) guidelines [4]. Likewise, numerous human studies using different strains of probiotics have reported probiotics to be effective in improving the clinical signs of AD in young children [8-10]. Although the exact mechanism of the immunomodulatory effect of probiotics in human and canine AD has not been fully understood, a close relationship between intestinal bacterial flora and the numerous factors associated with the pathogenesis of $\mathrm{AD}$ has been suggested [11]. 
The main probiotics used for the treatment of human atopic patients are mainly Lactobacilli and Bifidobacteria. This is because the number of Lactobacilli and Bifidobacteria is significantly lower in the fecal samples of human infants and children with $\mathrm{AD}$ than in healthy individuals [12]. A study of human $\mathrm{AD}$ reported that pruritus and medication scores were improved by the administration of probiotics consisting of Lactobacilli strain [13]. Another study also reported great improvements in the clinical signs in atopic patients after combined administration of Bifidobacteria stain [10]. However, there have been few studies on the therapeutic or complementary effects of probiotics, especially Bifidobacterium strain, in canine AD. Therefore, we aimed to evaluate the beneficial effects of oral administration of Bifidobacterium (B.) longum in dogs with $\mathrm{AD}$.

\section{Materials and Methods}

\section{Animals}

We enrolled 11 client-owned dogs that had been diagnosed with mild to moderate $\mathrm{AD}$ and treated according to ICADA guidelines [4] at the Veterinary Teaching Hospital of Chungbuk National University (Cheongju, South Korea). The diagnostic criteria for canine $\mathrm{AD}$ were based on the medical history, typical clinical signs according to the ICADA guidelines [4], fulfillment of more than 5 postulates in Favrot's criteria [14], and/or a positive intradermal skin test. To rule out cutaneous adverse food reactions, we performed a diet elimination trial for at least 8 weeks using a commercial hydrolyzed hypoallergenic diet, Royal Canin Hypoallergenic and Anallergenic (Royal Canin; Aimargues, France). We ruled out the possibility of other diseases that can cause pruritus and skin lesions through proper tests and treatments. The inclusion criteria included: a minimum score of 3 in the pruritus visual analog scale (PVAS) or of 30 in the Canine Atopic Dermatitis Extent and Severity Index (CADESI)-4 $[15,16]$.

The dogs were randomly allocated to 2 groups; namely, the probiotics and control group. The probiotics group $(n=7)$ was prescribed with $B$. longum while the control group $(n=4)$ received a placebo powder. All formerly prescribed drugs for controlling canine $\mathrm{AD}$ were continued during the study period. We obtained informed consent from all the owners prior to the experiment.

\section{Administration of probiotics}

We obtained B. longum from the National Institute of Animal Science, Rural Development Administration. It had been isolated from feces of healthy Korean neonates and anaerobically cultured $\left(5 \times 10^{10}\right.$ colony-forming units per $\left.2 \mathrm{~g}\right)$. Probiotics in sachets were stored at $4^{\circ} \mathrm{C}$ and orally administered to the dog once a day for 12 consecutive weeks.

\section{Assessments}

Assessment of the CADESI-4 score, transepidermal water loss (TEWL), and medication score was conducted by veterinarians while that of PVAS was performed by the dog-owners. All clinical indices were surveyed every 4 weeks starting from the baseline to the 12 weeks.

\section{PVAS}

The severity of pruritus was graded using the PVAS score [16]. The PVAS values were determined based on historytaking and evaluation by the owners. The PVAS consists of 10 scales with 0 indicating no pruritus and 10 indicating the most severe pruritus.

\section{CADESI-4}

The extent and severity of skin lesions were evaluated using the CADESI-4, which was designed to assess 20 different body parts. We assessed 3 skin lesions including erythema, lichenification, and excoriation/alopecia based on a four-point severity scale. The scale indicated none (score 0 ), mild (score 1), moderate (score 2), or severe (score 3). We assessed 20 body sites, 3 types of lesion, and 4 grades of severity, and thus generating a maximal score of $20 \times$ $3 \times 3=180$. The CADESI- 4 cut-off scores for mild, moderate, and severe $\mathrm{AD}$ were 10,35 , and 60 , respectively [15].

\section{TEWL}

Prior to assessing TEWL, the dogs were allowed to acclimate to the environment of the test room. We measured TEWL using an unventilated closed-chamber device, VaporMeter SWL3 (Delfin Technologies Ltd., Kuopio, Finland) according to the manufacturer's instructions. To minimize the TEWL variability, we performed five consecutive measurements for 10 seconds at a time on both pinnae, axillae, and the groin in a constant environment (ambient temperature: $24-25^{\circ} \mathrm{C}$; relative humidity: 44-66\%). Subsequently, we excluded the maximum and minimum values and averaged the remaining results and presented the average as $\mathrm{g} / \mathrm{m}^{2} / \mathrm{h}$.

\section{Medication score}

Medication score used in this study was based on previously described [7] and we used modified version. Alteration of consumed doses of the drugs including prednisolone, oclacitinib, and ciclosporin was calculated and converted into a medication score. A medication score of 1.0 was assigned to the drug dose that was being administered prior to the start of probiotics administration.

\section{Statistical analysis}

Data were analyzed using GraphPad Prism 7 software (GraphPad Software Inc., San Diego, CA, USA). The Wilcoxon test was conducted to analyze the PVAS, CADESI-4, TEWL, and medication scores in each group. The betweengroup differences of the values obtained every 4 weeks were also analyzed using the non-parametric Mann-Whitney test. All data were expressed as mean \pm standard deviation (SD). Differences were considered statistically significant at $p<0.05$. 

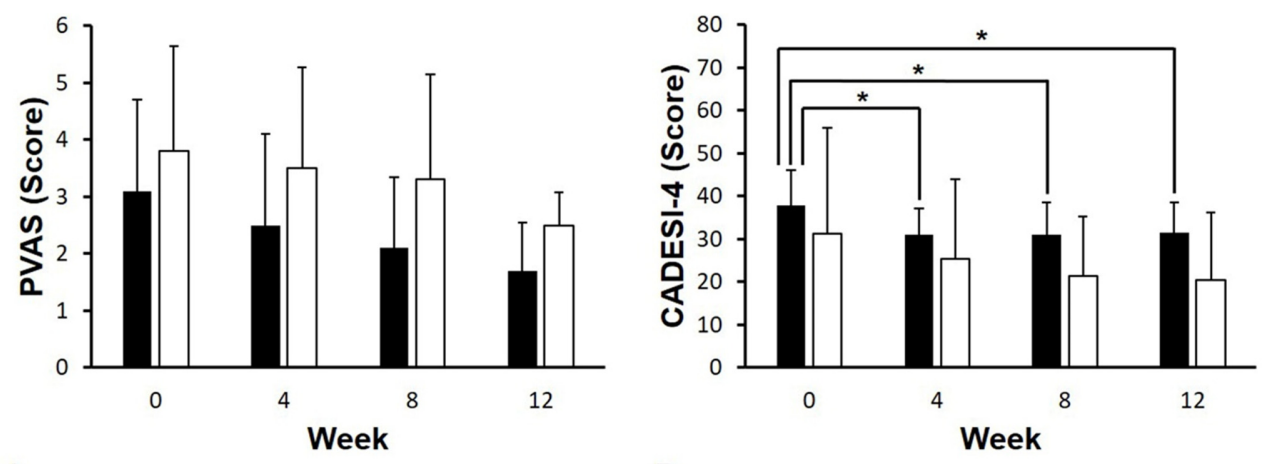

A

- Probiotics group $\square$ Control group

B
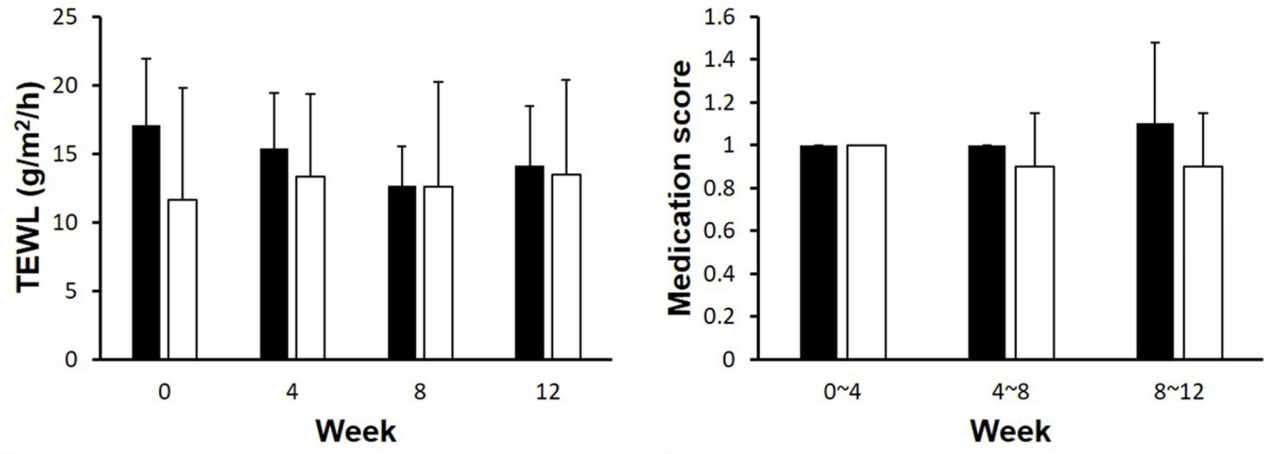

C

- Probiotics group $\square$ Control group

- Probiotics group $\square$ Control group

Fig. 1. Time-course changes in the pruritus visual analog scale (PVAS) (A), Canine Atopic Dermatitis Extent and Severity Index (CADESI)-4 (B), transepidermal water loss (TEWL) (C), and medication score (D) in the probiotics and control groups. (A, C, D) There were no statistically significant between-group differences at each time point in the PVAS, TEWL, and medication scores. There were also no significant within-group differences at all time-points. (B) There was a significant decrease in the CADESI-4 scores in the probiotics group at 4,8 , and 12 compared to the baseline; however, there was no significant difference in the control group. There was no significant between-group difference at each time point. ${ }^{*} p<0.05$ compared to the baseline. Error bars indicate the mean \pm standard deviation.

\section{Evaluation for safety}

Adverse effects of the probiotics were monitored by the dog-owners at home and by the veterinarians at every visit.

\section{Results}

\section{Animal population}

The signalments (age, sex, and breed) and the previously prescribed drugs and their dosage in the probiotics $(n=7)$ and control $(n=4)$ group are shown in Table 1 . In the probiotics group, the major dog breeds were Shih Tzu $(n=2)$ and Maltese $(n=2)$. However, in the control group, there was no major dog breed. The mean ages in the probiotics and control group were $6.29 \pm 3.35$ and $7 \pm 5.10$ years old (mean \pm $\mathrm{SD})$, respectively. All the 11 enrolled dogs were treated once or twice daily using oclacitinib $(0.4 \mathrm{mg} / \mathrm{kg})$ (Apoquel ${ }^{\circledR}$; Zoetis Inc., Florham Park, NJ, USA) prior to the start of the experiment.

\section{PVAS}

Although the PVAS scores in both groups consistently decreased until the end of the experiment, there were no significant within-group differences at all time-points (Fig. 1A). Further, there were no significant between-group differences at each time point (baseline, $p=0.694 ; 4$ weeks, $p=0.280 ; 8$ weeks, $p=0.336$; 12 weeks, $p=0.197$ ).

\section{CADESI-4}

Compared to the baseline scores, there was a significant decrease of the CADESI-4 scores at 4,8 , and 12 weeks in the probiotics group ( $0-4$ weeks, $p=0.02 ; 0-8$ weeks, $p=$ 0.03 ; $0-12$ weeks, $p=0.02$ ) (Fig. 1B). However, there was no significant between-group difference at each time point $(0$ week, $p=0.824 ; 4$ weeks, $p=0.715 ; 8$ weeks, $p=0.291 ; 12$ weeks, $p=0.382$ ).

\section{TEWL}

There was no significant between-group difference at each time point (baseline, $p=0.158$; 4 weeks, $p=0.158$; 8 weeks, $p=0.842 ; 12$ weeks, $p>0.999)$. In addition, there was no evident effect of the probiotics and placebo powder on TEWL within each group over time (Fig. 1C). 
Table 1. Summary of signalments and previously prescribed drugs of the 11 dogs involved in the study

\begin{tabular}{|c|c|c|c|c|}
\hline Patient No. & Age (yr) & Sex & Breed & Previously prescribed drugs \\
\hline $\mathrm{P}-1$ & 10 & IF & Shih Tzu & Oclacitinib $0.4 \mathrm{mg} / \mathrm{kg}$ twice daily \\
\hline P-2 & 5 & $\mathrm{CM}$ & Cocker Spaniel & Oclacitinib $0.4 \mathrm{mg} / \mathrm{kg}$ once daily \\
\hline P-3 & 8 & $\mathrm{CM}$ & Maltese & Oclacitinib $0.4 \mathrm{mg} / \mathrm{kg}$ twice daily \\
\hline P-4 & 3 & $\mathrm{SF}$ & Maltese & Oclacitinib $0.4 \mathrm{mg} / \mathrm{kg}$ once daily \\
\hline P-5 & 3 & $\mathrm{CM}$ & French Bulldog & Oclacitinib $0.4 \mathrm{mg} / \mathrm{kg}$ twice daily \\
\hline P-6 & 4 & SF & Pekingese & Oclacitinib $0.4 \mathrm{mg} / \mathrm{kg}$ once daily \\
\hline P-7 & 11 & SF & Shih Tzu & Oclacitinib $0.4 \mathrm{mg} / \mathrm{kg}$ once daily \\
\hline $\mathrm{C}-1$ & 2 & $\mathrm{CM}$ & Boston Terrier & Oclacitinib $0.4 \mathrm{mg} / \mathrm{kg}$ twice daily \\
\hline $\mathrm{C}-2$ & 14 & SF & Shih Tzu & Oclacitinib $0.4 \mathrm{mg} / \mathrm{kg}$ once daily \\
\hline $\mathrm{C}-3$ & 5 & $\mathrm{SF}$ & Maltese & Oclacitinib $0.4 \mathrm{mg} / \mathrm{kg}$ twice daily \\
\hline $\mathrm{C}-4$ & 7 & $\mathrm{CM}$ & Dachshund & Oclacitinib $0.6 \mathrm{mg} / \mathrm{kg}$ once daily \\
\hline
\end{tabular}

P, probiotics group; C, control group; CM, castrated male; IF, intact female; SF, spayed female.

\section{Medication score}

Regarding the medication score, there was a slight increase at 8-12 weeks in the probiotics group and a slight decrease at 4-8 weeks in the control group; however, there were no significant within-group differences at all time-points $(p>0.99)$. Moreover, there were no significant between-group differences at each time point $(p>0.99)$.

\section{Evaluation for safety}

No adverse effects were reported during the 12 weeks and after the experimental period in both the probiotics and control groups.

\section{Discussion}

Several studies have reported beneficial effects of probiotics in the prevention and management of $\mathrm{AD}$ in human patients. Specifically, a study reported that infants of mothers who had received the probiotics had a reduced incidence rate of $\mathrm{AD}$ compared to the control group [17]. Further, administration of probiotics to children aged 6 months to 13 years and with moderate or severe $\mathrm{AD}$ has been reported to moderately improve the clinical severity of $\mathrm{AD}[8,9]$. In adult $\mathrm{AD}$ patients, there have been reported improvements of cutaneous symptoms, intestinal flora in the feces, and patients' psychological status [10]. There have also been several studies using mouse model of AD. In these studies [1820], probiotics prevented the development of $\mathrm{AD}$ and allergic march, progression of allergic disorders from $\mathrm{AD}$, by inhibition of inflammatory cytokines.

In veterinary medicine, there have been few reports on the effects of probiotics on canine AD. Moreover, ICADA has suggested that there is currently insufficient evidence on the clinical basis for the administration of oral probiotics as nonspecific immunotherapy to treat or prevent canine AD [4]. However, in the last decade, several studies on the complementary effects of Lactobacilli on canine AD have been performed [5-7]. They indicated that probiotics showed comple- mentary benefits for dogs with $\mathrm{AD}$ who had been managed with conventional treatment. Three of these studies were based on the experimental AD model sensitized by house dust mite and only one study on Lactobacillus paracasei $\mathrm{K} 71$ performed actual veterinary clinical trials. Although it is already known that the beneficial effects of probiotics are greatly dependent on the specific strains, e.g., Lactobacilli and Bifidobacteria [21], there has been no study on the effect of Bifidobacteria on canine AD.

Intestinal bacterial flora is important not only in retaining the structural and functional integrity of the intestine but also in immune system regulation $[22,23]$. There have been several attempts to use probiotics to control the canine intestinal bacterial flora since intestinal microbiota imbalance may affect the gut as well as extra-intestinal organ systems such as the skin $[24,25]$. The specific mechanisms through which the gut microbiota produces its effects are beginning to be understood with some studies reporting a close correlation between intestinal microflora and numerous factors associated with the pathophysiology of $\mathrm{AD}$, such as immunity and inflammation [26,27].

Based on the results of the study using Lactobacilli strain [7], we examined the hypothesis that probiotics have a beneficial effect on the alleviation of canine AD using a different strain of probiotics, i.e., Bifidobacteria, instead of Lactobacilli. However, in this randomized, placebo-controlled study, we found clinical complementary effects of the addition of probiotics for controlling the severity of canine AD by alleviating skin lesions. We found that the CADESI-4 scores of the probiotics group significantly decreased from the baseline to 4, 8 and 12 weeks; however, this was not observed in the control group. These findings are consistent with those of a study evaluating the complementary effects of Lactobacillus paracasei $\mathrm{K} 71$ on canine AD [7]. TEWL in the probiotics group showed a similar tendency; however, it did not reach statistical significance within the group. Therefore, it appears that B. longum might have supplementary effect of only improving skin lesions in dogs with $\mathrm{AD}$ receiving standard 
therapy. However, this effect might be fairly restricted given that there was no statistically significant difference compared to the control group in the CADESI-4 scores after initiation of administration.

Although many human studies have reported a beneficial effect of probiotics in not only improving clinical signs of $\mathrm{AD}$ but also reducing the incidence of $\mathrm{AD}$, other studies have reported that probiotics supplementation neither improved the severity of AD nor reduced the incidence of $\mathrm{AD}[28,29]$.

A possible association between probiotics use and some adverse effects in humans has been reported. Further, theoretical side effects such as systemic infections, deleterious metabolic activities, excessive immune stimulation in susceptible individuals, gene transfer, and minor gastrointestinal symptoms have been reported [30]. However, in this study, no adverse effects of probiotics were reported by the dogs-owners and veterinarians.

The limitation of this study is the relatively small size of dogs. Although we did not find a significant between-group difference in the clinical signs, studies with larger samples are needed to further evaluate the effect of probiotics. Moreover, since several studies have reported that administration of probiotics prevented the development of $\mathrm{AD}$ in mouse model [18-20], further research is needed to evaluate the effect of probiotics on pregnant and young adult dogs. Further, future studies should consider stopping the use of drugs for canine AD treatment so as to ideally identify the beneficial effects of probiotics. In this study, it was difficult for the dog-owners to visit the veterinary hospital without any medication for 12 weeks. Therefore, the dogs in this study were maintained with a minimum dose of medication to manage the clinical signs of AD.

In conclusion, although it was not sufficient to reduce pruritus, TEWL, and the dosage of prescribed drugs for managing canine $\mathrm{AD}$, our preliminary results showed that oral administration of B. longum was effective in improving skin lesions and could be considered as a supportive therapy for canine AD.

\section{Acknowledgement}

This work was carried out with the support of "Cooperative Research Program for Agriculture Science and Technology Development (Project No: PJ01283408)" Rural Development Administration, Republic of Korea.

\section{References}

1. Halliwell R. Revised nomenclature for veterinary allergy. Vet Immunol Immunopathol 2006;114:207-208.

2. Bizikova P, Pucheu-Haston CM, Eisenschenk MN, Marsella R, Nuttall T, Santoro D. Review: Role of genetics and the environment in the pathogenesis of canine atopic dermatitis. Vet Dermatol 2015;26:95-e26.

3. Hillier A, Griffin CE. The ACVD task force on canine atopic dermatitis (I): incidence and prevalence. Vet Immunol
Immunopathol 2001;81:147-151.

4. Olivry T, DeBoer DJ, Favrot C, Jackson HA, Mueller RS, Nuttall $\mathrm{T}$, Prélaud $\mathrm{P}$; International Committee on Allergic Diseases of Animals. Treatment of canine atopic dermatitis: 2015 updated guidelines from the International Committee on Allergic Diseases of Animals (ICADA). BMC Vet Res 2015; 11:210-224.

5. Marsella R. Evaluation of Lactobacillus rhamnosus strain GG for the prevention of atopic dermatitis in dogs. Am J Vet Res 2009;70:735-740.

6. Marsella R, Santoro D, Ahrens K. Early exposure to probiotics in a canine model of atopic dermatitis has long-term clinical and immunological effects. Vet Immunol Immunopathol 2012;146:185-189.

7. Ohshima-Terada Y, Higuchi Y, Kumagai T, Hagihara A, Nagata M. Complementary effect of oral administration of Lactobacillus paracasei K71 on canine atopic dermatitis. Vet Dermatol 2015;26:350-353.

8. Rosenfeldt V, Benfeldt E, Nielsen SD, Michaelsen KF, Jeppesen DL, Valerius NH, Paerregaard A. Effect of probiotic Lactobacillus strains in children with atopic dermatitis. J Allergy Clin Immunol 2003;111:389-395.

9. Weston S, Halbert A, Richmond P, Prescott SL. Effects of probiotics on atopic dermatitis: a randomised controlled trial. Arch Dis Child 2005;90:892-897.

10. Yoshida Y, Seki T, Matsunaka H, Watanabe T, Shindo M, Yamada N, Yamamoto O. Clinical effects of probiotic Bifidobacterium breve supplementation in adult patients with atopic dermatitis. Yonago Acta Med 2010;53:37-45.

11. Craig JM. Atopic dermatitis and the intestinal microbiota in humans and dogs. Vet Med Sci 2016;2:95-105.

12. Björkstén B, Sepp E, Julge K, Voor T, Mikelsaar M. Allergy development and the intestinal microflora during the first year of life. J Allergy Clin Immunol 2001;108:516-520.

13. Yamamoto K, Yokoyama K, Matsukawa T, Kato S, Kato S, Yamada K, Hirota T. Efficacy of prolonged ingestion of Lactobacillus acidophilus L-92 in adult patients with atopic dermatitis. J Dairy Sci 2016;99:5039-5046.

14. Hensel P, Santoro D, Favrot C, Hill P, Griffin C. Canine atopic dermatitis: detailed guidelines for diagnosis and allergen identification. BMC Vet Res 2015;11:196.

15. Olivry T, Saridomichelakis M, Nuttall T, Bensignor E, Griffin CE, Hill PB; International Committe on Allergic Diseases of Animals (ICADA). Validation of the Canine Atopic Dermatitis Extent and Severity Index (CADESI)-4, a simplified severity scale for assessing skin lesions of atopic dermatitis in dogs. Vet Dermatol 2014;25:77-85.

16. Rybnícek J, Lau-Gillard PJ, Harvey R, Hill PB. Further validation of a pruritus severity scale for use in dogs. Vet Dermatol 2009;20:115-122.

17. Huurre A, Laitinen K, Rautava S, Korkeamäki M, Isolauri E. Impact of maternal atopy and probiotic supplementation during pregnancy on infant sensitization: a double-blind placebocontrolled study. Clin Exp Allergy 2008;38:1342-1348.

18. Kim HJ, Kim YJ, Kang MJ, Seo JH, Kim HY, Jeong SK, Lee SH, Kim JM, Hong SJ. A novel mouse model of atopic dermatitis with epicutaneous allergen sensitization and the effect of Lactobacillus rhamnosus. Exp Dermatol 2012;21: 672-675.

19. Kim HJ, Kim HY, Lee SY, Seo JH, Lee E, Hong SJ. 
24 Kang-Il Lee, Taesik Yun, Junsang Ham, Wan-Kyu Lee, Ji-Houn Kang, Mhan-Pyo Yang, Byeong-Teck Kang

Clinical efficacy and mechanism of probiotics in allergic diseases. Korean J Pediatr 2013;56:369-376.

20. Kim HJ, Kim YJ, Lee SH, Yu J, Jeong SK, Hong SJ. Effects of Lactobacillus rhamnosus on allergic march model by suppressing Th2, Th17, and TSLP responses via $\mathrm{CD}^{+} \mathrm{CD} 25^{+} \mathrm{Foxp}^{+}$Tregs. Clin Immunol 2014;153:178-186.

21. Larsen N, Vogensen FK, Gøbel R, Michaelsen KF, Abu AlSoud W, Sørensen SJ, Hansen LH, Jakobsen M. Predominant genera of fecal microbiota in children with atopic dermatitis are not altered by intake of probiotic bacteria Lactobacillus acidophilus NCFM and Bifidobacterium animalis subsp. lactis Bi-07. FEMS Microbiol Ecol 2011;75:482-496.

22. Furusawa Y, Obata Y, Fukuda S, Endo TA, Nakato G, Takahashi D, Nakanishi Y, Uetake C, Kato K, Kato T, Takahashi M, Fukuda NN, Murakami S, Miyauchi E, Hino S, Atarashi K, Onawa S, Fujimura Y, Lockett T, Clarke JM, Topping DL, Tomita M, Hori S, Ohara O, Morita T, Koseki H, Kikuchi J, Honda K, Hase K, Ohno H. Commensal microbe-derived butyrate induces the differentiation of colonic regulatory T cells. Nature 2013;504:446-450.

23. Purchiaroni F, Tortora A, Gabrielli M, Bertucci F, Gigante G, Ianiro G, Ojetti V, Scarpellini E, Gasbarrini A. The role of intestinal microbiota and the immune system. Eur Rev Med Pharmacol Sci 2013.17:323-333.

24. Sekirov I, Russell SL, Antunes LC, Finlay BB. Gut microbiota in health and disease. Physiol Rev 2010;90:859-904.

25. Suchodolski JS, Simpson K. Canine gastrointestinal microbiome in health and disease. Veterinary Focus 2013;23:22-28.

26. Belkaid Y, Hand TW. Role of the microbiota in immunity and inflammation. Cell 2014;157:121-141.

27. Bowe WP, Logan AC. Acne vulgaris, probiotics and the gutbrain-skin axis - back to the future? Gut Pathog 2011;3:1.

28. Brouwer ML, Wolt-Plompen SA, Dubois AE, van der Heide S, Jansen DF, Hoijer MA, Kauffman HF, Duiverman EJ. No effects of probiotics on atopic dermatitis in infancy: a randomized placebo-controlled trial. Clin Exp Allergy 2006; 36:899-906.

29. Kopp MV, Hennemuth I, Heinzmann A, Urbanek R. Randomized, double-blind, placebo-controlled trial of probiotics for primary prevention: no clinical effects of Lactobacillus GG supplementation. Pediatrics 2008;121:e850-e856.

30. Doron S, Snydman DR. Risk and safety of probiotics. Clin Infect Dis 2015;60 Suppl 2:S129-S134. 